\title{
The effect of Hbs on accuracy of pulse oximetry in blood oxygen saturation level measurement among adult patients with sickle cell disease
}

\author{
Esmaeil Sadeghi Poura, Saeed Hayatib, Mohammad Javad Khorasanic, Seyed \\ Ashkan Tabibzadeh Dezfulib,* \\ ${ }^{a}$ Emergency Medicine Specialist, Hormozgan University of Medical Sciences, Bandar Abbas, Iran \\ ${ }^{b}$ Trauma and Emergency Medicine Research Center, Hormozgan University of Medical Sciences, \\ Bandar Abbas, Iran \\ ${ }^{c}$ Chairman of Emergency Medicine, Hormozgan University of Medical Sciences, Bandar Abbas, Iran
}

Received: 12 September 2019, Accepted: 15 November 2019, Published: 02 December 2019

\begin{abstract}
This study was conducted to investigate the effect of Hbs on accuracy of pulse oximetry in blood oxygen saturation level measurement among adult patients with sickle cell disease crises in Shahid Mohammadi Hospital of Bandar Abbas during 2017. For this cross-sectional study, the statistical population included all patients with sickle cell disease who were hospitalized in emergency department of Shahid Mohammadi Hospital of Bandar Abbas during the second half of 2017. At first, pulse oximetry was performed on subjects and then the arterial blood samples were taken to analyze arterial blood gas (ABG) according to the protocol. Blood samples were taken from each subject for electrophoresis and determination of $\mathrm{Hbs}$ level. The mean result of pulse oximetry on patients was $97.69 \pm 1.57$, with the lowest $90 \%$ and the highest $98 \%$. In contrast, the oxygen content of the ABG analysis varied from 78 to $93 \%$, and the mean of these results was $87.54 \pm 3.54$. The $\mathrm{Hbs}$ level of electrophoresis on patients was $72.78 \pm 8.12 \%$. The difference between the blood oxygen saturation level in the blood obtained by pulse oximetry and arterial blood gas analysis was less than $10 \%$. The findings showed that the oxygen saturation level in the blood measured using two techniques of pulse oximetry and AGB analysis was significantly different. It is advisable to use more accurate techniques to estimate the oxygen saturation level in the blood and to determine the rate of hypoxia.
\end{abstract}

Keywords: Hemoglobin S; pulse oximetry; sickle cell; saturation O2.

\section{Introduction}

Sickle cell disease (SCD) is an autosomal recessive disorder due to inheritance of a type of $\beta$-globin called sickle hemoglobin (Hbs). Carriers of this gene are resistant to malaria infection, hence the disease has a significant incidence in areas with high prevalence of malaria $[2,3]$. The prevalence of this disease has been reported to be high in the southern regions of Iran, especially in the Persian Gulf [4]. In people with sickle cell

*Corresponding author: Seyed Ashkan Tabibzadeh Dezfuli 
disease, a significant rate of illness and mortality can be observed, among which severe anemia, stroke, acute chest pain, pain attack, spleen and kidney failure, and sensitivity to infections can be mentioned [1,5]. In disease crises, dysfunction occurs in red blood cells, which is due to hypoxia [6]. For this reason, accurate measurements of oxygen saturation level in the blood in these patients, especially in emergency cases in which the patient is hospitalized because of pain crisis, is of great importance [7]. Sickle cell disease is a group of disorders that affects hemoglobin, the molecule in red blood cells that delivers oxygen to cells throughout the body. The symptoms of SCD usually begin in childhood and characteristic features of this disorder include a low number of red blood cells (anemia), repeated infections, and periodic episodes of pain [8]. Hypoxemia is one of the common complications of $\mathrm{SCD}$, which is due to cyclization of red blood cells and vascular obstruction, leading to tissue hypoxia and ultimately pain crisis in these patients [8,9]. In each crisis, the obstruction created in small vessels may cause micro-infarcts in any organ, especially the lungs, resulting their dysfunction. On the one hand, the pulmonary involvement increases the risk of hypoxia in these patients $[7,10]$. Hypoxia causes the onset of cyclization of red blood cells, so correcting it plays a key role in improving the patient's condition $[6,11]$.

Techniques available to measure the oxygen saturation level for the evaluation of hospitalized patients include pulse oximetry and arterial blood gas analysis (ABG) [12-14]. The pulse oximetry is a simple, non-invasive and fast technique [15]. However, in patients with abnormal hemoglobin, it may not indicate a proper oxygen saturation level [16-18]. In contrast, the arterial blood gas
(ABG) analysis, which is an invasive technique, measures the oxygen saturation level in the blood regardless of the content of hemoglobin $[19,20]$. An accurate examination of hypoxemia in patients with SCD and the selection of the most appropriate technique with the least error makes it possible for these patients to be better evaluated during the crisis [21] and appropriate treatment for these patients to be selected [22]. Studies to evaluate the proper techniques for measuring oxygen saturation level in the blood of patients with SCD provide conflicting results [23] among which, the results of studies by Dr. Cres et al., Dr. Ortiz et al., and Dr. Ogah et al., showed that patients with SCD during hospitalization due to the crisis exhibited similar results using pulse oximetry and ABG analysis techniques [8,11, and 24]. In contrast, the results of a study conducted by Dr. Comber and Lopez suggested that oximetry pulses showed less oxigenisation in patients with SCD in crisis than in the $A B G$ analysis $[25,26]$. The aim of the present study was investigating the effect of $\mathrm{Hbs}$ on accuracy of pulse oximetry in blood oxygen saturation level measurement among adult patients with sickle cell disease crises in Shahid Mohammadi Hospital of Bandar Abbas.

\section{Methodology}

This was a descriptive-analytic crosssectional study conducted and aimed at investigating the effect of $\mathrm{Hbs}$ on accuracy of pulse oximetry in blood oxygen saturation level measurement among adult patients with sickle cell disease crises in Shahid Mohammadi Hospital of Bandar Abbas during the second 6 months of 2017. This study was approved by the ethics committee of Shahid Mohammadi Hospital of Bandar Abbas. The statistical population included all patients with complaints of SDS crisis who were hospitalized in 
emergency department of Shahid Mohammadi Hospital of Bandar Abbas during the second half of 2017. At first, pulse oximetry was performed on subjects and the results were included in the relevant data collection form. The arterial blood samples were then taken to analyze arterial blood gas (ABG) according to the protocol and transferred to the laboratory of Shahid Mohammadi Hospital in Bandar Abbas. The relevant results were then recorded in data collection form for subjects. Blood samples were taken from each subject for electrophoresis and determination of Hbs level. The results were recorded in the data collection forms after a laboratory examination.

\section{Studied patients}

In this study, 91 patients with complaints of SDS crisis who were referred to emergency department of Shahid Mohammadi Hospital of Bandar Abbas during the second half of 2017 were examined. This sample was selected by census method and all patients who were willing to participate at the time of study were included. Inclusion criteria included informed consent of the patient to participate in the study. Patients who did not want to participate in the study and did not complete the written consent were excluded. Other exclusion criteria were skin pigmentation, nail polish, venous pulse, severe anemia ( $\mathrm{hb}<5$ ), vascular dislocation, low blood pressure, and fever that disrupts the pulse oximetry. Subsequently, the demographic data of each patient were collected during oral interviews or using the data contained in the patients' records and entered into the patient's data collection form. Then, pulse oximetry was performed on subjects and the results were recorded in the relevant data collection form. At the same time, the taken arterial blood samples were transferred to the laboratory of Shahid
Mohammadi Hospital in Bandar Abbas in order to analyze the blood gases under the sample storage conditions. The samples were also sent to the lab for electrophoresis and determination of $\mathrm{Hbs}$ level. Finally, the results were recorded in the data collection form for each subject. In this study, the data were collected through oral interview with patients, results displayed by pulse oximeter, results of $\mathrm{ABG}$ analysis and electrophoresis. Pulse oximetry was performed on each patient using Masimu technology and the results were recorded in the data collection form for that patient.

\section{Statistical analysis}

The data were analyzed by SPSS version 19 using regression models. The accuracy and correlation of pulse oximetry and ABG were investigated with kappa coefficient. The comparisons between groups were analyzed by t-test. A value of $\mathrm{P}<0.05$ was considered to be statistically significant.

\section{Results and discution}

The results of pulse oximetry, ABG analysis and electrophoresis were analyzed after being collected. The mean age of patients was $35.8 \pm 9.1$ years, of which $67.4 \%$ were male. The mean result of pulse oximetry on patients was 97.69 \pm 1.57 , with the lowest $90 \%$ and the highest $98 \%$. In contrast, the oxygen content of the ABG analysis varied from 78 to $93 \%$, and the mean of these results was $87.54 \pm 3.54$. The mean difference between the oxygen saturation level in the blood from pulse oximetry and ABG analysis was $7.14 \pm 2.24$. The minimum difference was $2 \%$ and the maximum difference was $14 \%$. The percent of error rate of the results derived by pulse oximetry and ABG analysis was on average $7.56 \pm 2.93$. The Hbs level of electrophoresis on patients was $72.78 \pm$ $8.12 \%$. The results indicated that the 
difference between the blood oxygen saturation level in the blood obtained by pulse oximetry and ABG analysis was less than $10 \%$. This difference was not related to the age and gender of the patients.

Table 1. Mean, standard deviation, minimum and maximum values of pulse oximetry and ABG analysis

\begin{tabular}{ccccc}
\hline & Mean & Standard Deviation & Maximum & Minimum \\
\hline Pulse oximetry & 94.69 & 90.00 & 98.00 & 1.57 \\
ABG & 87.54 & 78.00 & 93.00 & 3.54 \\
diff & 7.14 & 2.00 & 14.00 & 2.72 \\
Difference percentage & 7.56 & 2.11 & 15.22 & 2.93 \\
\hline
\end{tabular}

Table 2. The correlation between the Hbs level and the difference between the results of pulse oximetry and $\mathrm{ABG}$ analysis

\begin{tabular}{ccc}
\hline & & Diff \\
\hline \multirow{2}{*}{ Electrophoresis } & 0.047 & Pearson Correlation \\
& 0.662 & Sig. (2-tailed) \\
& 90 & $\mathrm{~N}$ \\
\hline
\end{tabular}

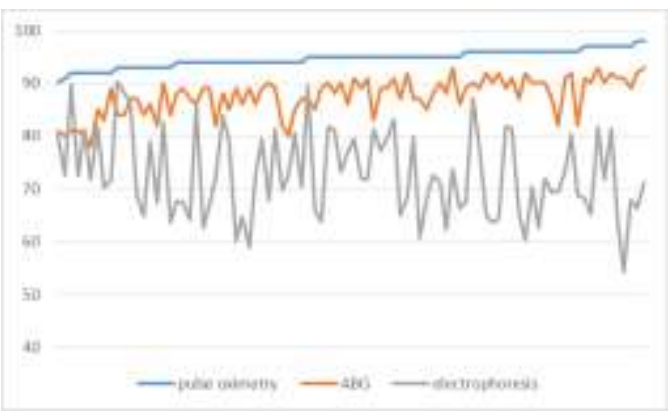

Figure 1. Sorting data by pulse oximetry

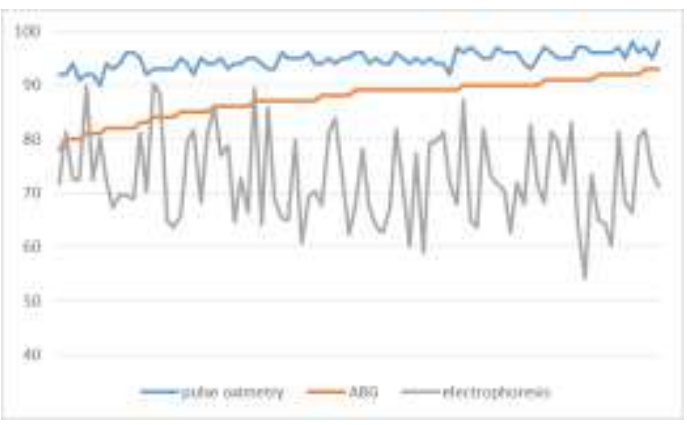

Figure 2. Sorting data by BAG analysis

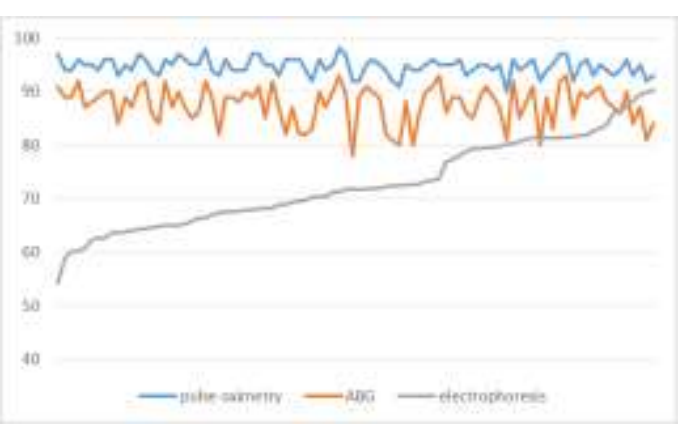

Figure 3. Sorting data by Hbs level
The correlation between the Hbs level and the difference between the results of pulse oximetry and $\mathrm{ABG}$ analysis was calculated using Pearson correlation coefficient. Since this value is close to zero, there is a weak and insignificant relationship $(\mathrm{P}$-value $=0.66)$ between the Hbs level and the results of pulse oximetry and ABG analysis. This relationship is also explored separately in the following three charts. In these three charts, each variable (pulse oximetry, ABG analysis, and Hbs level, respectively) is sorted from low to high, and the results of the other two variables are sorted in the same way. However, the ascending trend of no variable does not show any effect on the increase or decrease of the other two variables.

\section{Discussion}

The finding of the present study indicated that the saturated oxygen level in the blood was significantly different in measuring the use of two techniques of pulse oximetry and ABG analysis. There was no significant difference between the values obtained from these two techniques. These results were in contrast to the results of a study done by Dr. Cres et al. In the study of Dr. Cres 
conducted and aimed at determining the difference between the hemoglobin saturation level in patients with acute chest syndrome (ACS) and in patients with SCD using pulse oximetry and ABG analysis, the difference was $26.2 \pm 3.25$ that was reported to be negligible [25]. However, this value was about half the difference in the present study. Also, compared with the study of Dr. Ortiz et $a l$. , in which the difference of the oxygen saturation level in the blood using the two techniques on 22 patients was measured, the mean difference of the present study was 3.4 [25].

In a study by Dr. Needleman et al., who examined 16 patients with SCD, similar results were obtained. These results were significantly lower than the results obtained in this study [26]. However, in the studies by Dr. Ortiz and Dr. Needleman, a population of about one quarter of this study was investigated, which is one of the important limitations of these two studies [27,28]. In contrast, the results of the study were consistent with the results of the study by Dr. Comber. There was a significant difference between the results obtained from the two techniques. Despite the consistency of the results of the study by Dr. Fitzgerald and this study, the difference in the study by Dr. Fitzgerald was $21.5 \%$, which was three times more than the difference in the present study. However, in the study conducted by Dr. Fitzgerald, only 24 patients were examined [29]. The results of the study by Dr. Piansoni also suggested that in the treatment of patients with SCD, more reliable techniques than pulse oximetry should be used to estimate the oxygen saturation level in the blood [30].

\section{Conclusion}

The findings indicated that the oxygen saturation level in the blood measured by two techniques of pulse oximetry and
ABG analysis was significantly different. From the results of the present study, it can be concluded that for the treatment of patients with sickle cell disease, especially in the conditions of pain attack, it is advisable to use more accurate techniques to estimate the oxygen saturation level in the blood and to determine the rate of hypoxia.

\section{Limitations and recommendations}

The limitations were the sample storage and transfer conditions for ABG analysis, the distance between the laboratory and the emergency department, and the relative delay in obtaining the analyses answers for the necessary intervention on the patients. Future studies are recommended to examine the patient's condition during and after the pain attack in multi stages. It is recommended that patients aged less than 15 years are also examined.

\section{References}

[1] A. Ashley-Koch, Q. Yang, RS. Olney, Am. J. Epidem., 2000, 13, 839845.

[2] F.W. Lorey, J. Arnopp, G.C. Cunningham, Genet. Epidemiol., 1996, 13, 501-12.

[3] J.R. Aluoch. Trap. Med. Int. Health., 1997, 2, 568-571.

[4] K.h. Morad Zandian, M. Pedram, F. Kianpour Ghahfarokhi, Iran. J. Blood Cancer., 2009, 21, 55-57.

[5] J.A. Kark, D.M. Posey, H.R. Schumacher, New. Engl. J. Med., 1987, 317, 781-787.

[6] O.S. Platt, B.D. Thorington, D.J. Brambilla, New. Engl. J. Med., 1991, 325, 11-16.

[7] U.K. Dallas, A.H. Park, J. Null. Med., 1991, 13, 1617-1618.

[8] F.O. Ortiz, T.K. Aldriich, R.L. Nagel, L.J. Benjamin, Am. J. Resp. Critical Care Med., 1999, 17, 447-451.

[9] T.K. Aldrich, R.L. Nagel, Pulm. Critical. Care. Med., 1998, 7, 1-10; (B) 
N. Moradi kor, Int. J. Biosci, 2014, 4, 8999.

[10] O. Castro, D.J. Brambilla, B. Thorington, Blood., 1994, 84, 643-649.

[11] A.O. Ogah, A. Surat, A.G. Okoruwa, J. Ezeonwumelu, S.N. Okolo. Asian J. Med. Scie., 2012, 4, 161-165. [12] T. Neff, Chest., 1988, 21, 227-331.. [13] M.W. Wukitsch, M.T. Petterson, D.R. Tobler, J.A. Pologe, J. Clin. Monit., 1988, 4, 290-301.

[14] A.J. Williams, BMJ., 1998, 14, 317321.

[15] M. Verhovsek, M. Henderson, G. Cox, H. Luo, M. Steinberg, D. Chui, Am. J. Hem., 2010, 7, 882-885.

[16] R.J. Castle, U.P. Stroll, S. Chester, Arch. Intern. Med., 1989, 5, 185-188.

[17] G.D. Jay, F.E. Renzi, Ann. Emerg. Med., 1992, 17, 400-407; (b) N. Moradi kor, Euro. J. Exp. Bio, 2014, 4, 340-348. [18] T. Roman, H.P. Close, R. Cathcart, Am. J. Med., 1959, 9, 538-558.

[19] G. Thieme, K.G. Verlag, Klin. Padiatr., 2012, 224, 259-265.

[20] V.A. Forte, M.K. Malconian, R.L. Burse, J. Appl. Physiol., 1989, 67, 21352140.

How to cite this manuscript: Esmaeil Sadeghi Pour, Saeed Hayati, Mohammad Javad Khorasani, Seyed Ashkan Tabibzadeh Dezfuli. The effect of Hbs on accuracy of pulse oximetry in blood oxygen saturation level measurement among adult patients with sickle cell disease. Eurasian Chemical Communications, 2020, 2(3), 296-301. 